\title{
Laser dazzling of infrared focal plane array cameras
}

\section{Ric Schleijpen}

High-intensity irradiance effects have been evaluated as a countermeasure against detection and tracking with infrared camera systems.

Modern weapons systems often use infrared imaging for acquisition and tracking of targets. In the acquisition phase, the operator uses the system to detect the target and aim the weapon. In the tracking phase, the images from the camera are used to guide the weapon to the target. One possible method of protection against such imaging systems is laser dazzling. The intended effect is to deny the imager a clear view of the target by saturating the detector and consequently preventing the weapon from making a hit.

The problem of locating the threat and pointing the laser at it is closely connected to the countermeasure effectiveness. This has been investigated particularly for specific applications such as directed infrared countermeasure (DIRCM) systems for aircraft self-protection. Another application is sniper detection, which uses the 'cat's-eye' or retro-reflection effect, which occurs when a searching laser beam is directed at the visor of the rifle. We currently aim to use the retro-reflection effect in a wider range of applications for locating optics. ${ }^{1}$

The aim of our dazzling work is to quantify the effects of laser countermeasures on mid-wave infrared (MWIR) imaging to predict the decrease in the detection and pointing capabilities of weapons systems. Dazzling with a continuous or quasicontinuous pulsed laser causes a reversible saturation effect on the detector array. Most of the current MWIR lasers provide output power levels that do not exceed saturation levels for practical applications. Permanent damage effects require much higher irradiance levels at the camera front optics. This might be within reach at shorter ranges with these lasers, but is not addressed in our current research.

In our experiments we used a periodically poled lithium niobate optical parametric oscillator (PPLN OPO) laser pumped by an $\mathrm{Nd}: \mathrm{YVO}_{4}$ (yttrium orthovanadate) laser to generate a $3.9 \mu \mathrm{m}$ laser wavelength. ${ }^{2}$ Figure 1 presents images of a standard $256 \times 320$ indium antimonide $(\mathrm{InSb})$ camera irradiated by
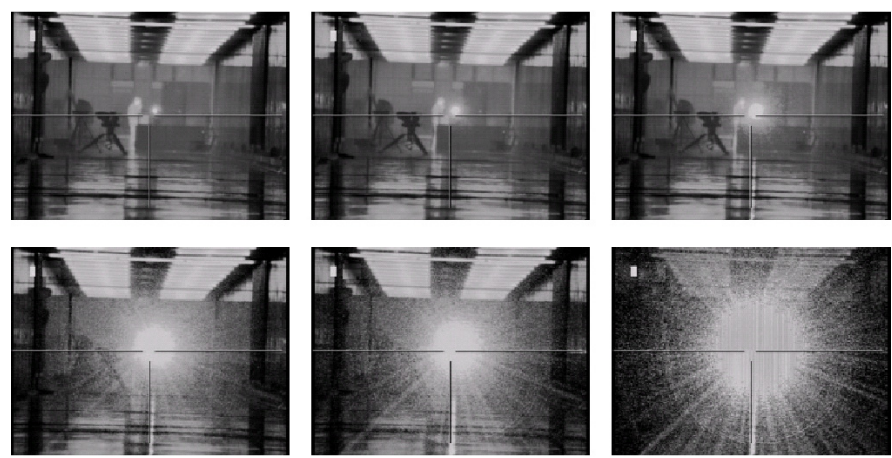

Figure 1. Impact of increasing laser irradiance on the InSb camera image.

the laser from a distance of $300 \mathrm{~m}$ at increasing power levels. We observe a saturated area, surrounded by an irregular scatter pattern that is fixed and whose effects increase with laser intensity. The growth of the central saturated area is purely related to optical effects and can be described mathematically using the diffraction-limited point spread function. Together with a method for estimating the threshold level for saturation of a single pixel, this relationship can be used to estimate the size of the saturated area as a function of laser power. ${ }^{3}$

A close look the enlarged camera image in Figure 2 reveals that for the highest laser irradiance levels, the saturated area no longer appears uniform in the central part of the dazzle area. The level of gray in the center is now lower than the maximum in the images for lower laser irradiance. This nonlinear saturation behavior seems to occur only for short laser pulse lengths on the order of 10ns. This became more apparent in experiments done in cooperation with colleagues in France and Germany using an imaging seeker simulator ${ }^{4}$ based on a cadmium mercury telluride (CMT) camera (see Figure 3). ${ }^{5}$ Irradiation of the camera with a continuous deuterium fluoride laser did not show the nonlinear effects. Applying the same average power in short laser pulses using a frequency-doubled carbon dioxide laser did 


\section{Newsroom}

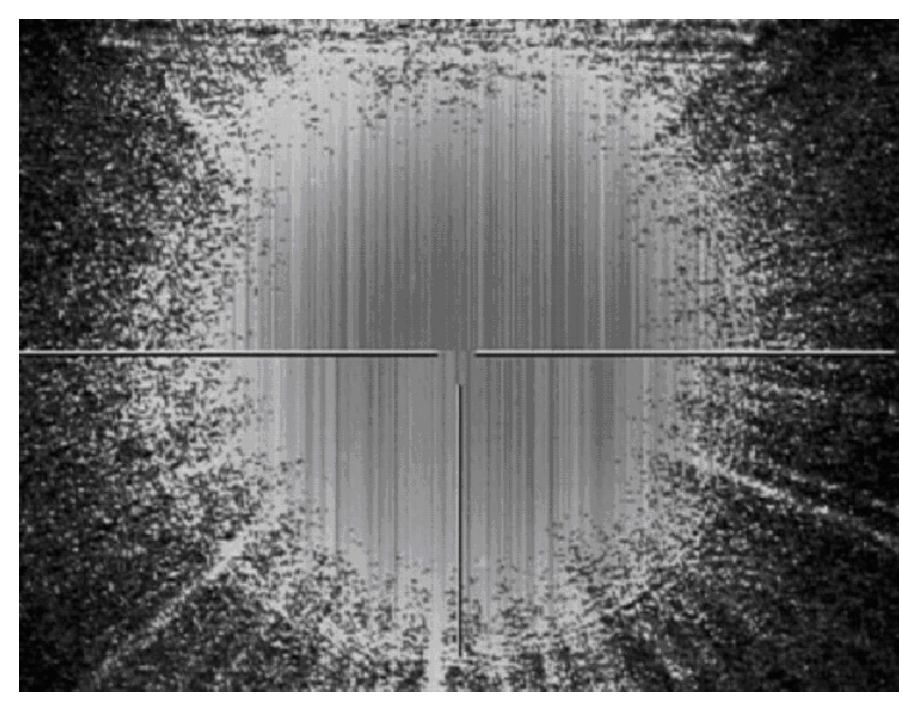

Figure 2. Observed laser dazzle image in the InSb camera, for maximum-power, short-pulse laser irradiance.

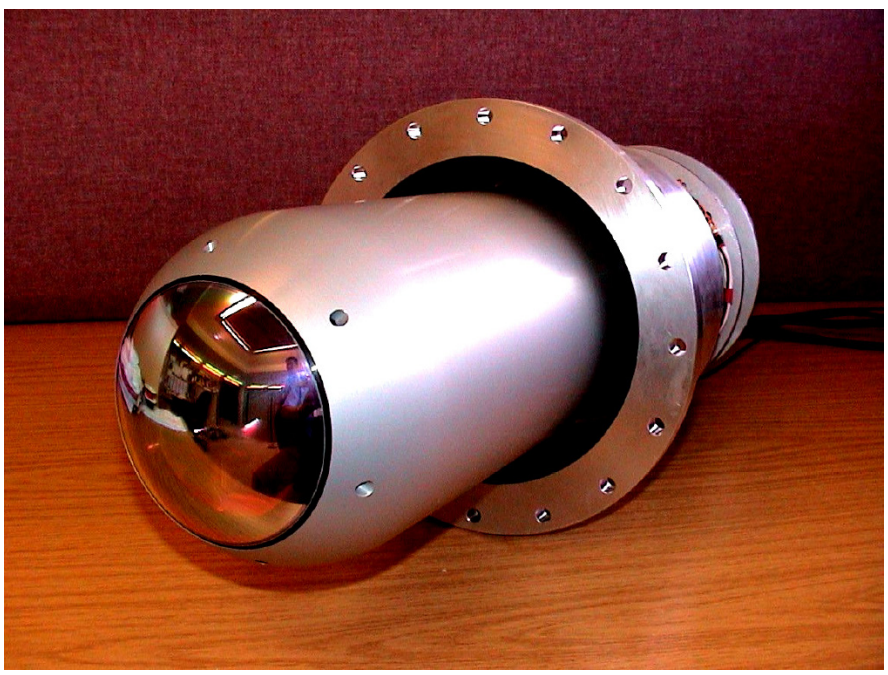

Figure 3. Imaging seeker simulator including the CMT camera.

cause nonlinear effects similar to the PPLN laser in the InSb camera. For both these lasers, the pulse lengths are approximately 10ns. As opposed to the saturation effects, which are caused by the limited charge storage capacity in the readout circuit, we attribute the nonlinear effects to the detector material itself. ${ }^{5}$

The nonlinear response of the detector to the high-power short laser pulses has consequences for the application of pulsed lasers in countermeasure systems. A countermeasure laser intends to generate a dazzling spot that is much brighter than the target signature. Because of the effects discussed here, at high power the intensity ratio between the dazzling spot and target is no longer proportional to the laser power, which means that the dazzling will be less effective than expected.

With laser dazzling, the size of the saturated area in the image is a measure of the laser-induced degradation of the target acquisition and tracking capability of the camera. Theoretical considerations and our experiments have resulted in a method to describe the size of the saturated area. Our experiments in a higher-power regime have shown a nonlinear detector response, which will complicate the prediction of the effectiveness of laser dazzling under these circumstances. Our current research is oriented to a better understanding of these nonlinear effects.

\section{Author Information}

\section{Ric Schleijpen}

TNO Defence, Security, and Safety

The Hague, The Netherlands

http:/ / www.tno.nl

Ric Schleijpen received both his MSc and PhD degrees in physics from the University of Technology in Eindhoven. In 1987 he joined TNO to enter the research field of infrared and ultraviolet detection, signatures, and countermeasures. From 2001 until 2007 he was program manager for Air Force electronic warfare research, focusing on infrared countermeasures. Since 2008 he has been running the joint electronic warfare research program at TNO, which combines the electronic weapons research at TNO for the Netherlands ministry of defense.

\section{References}

1. Arjan L. Mieremet and H. M. A. Schleijpen and Pierre-Nicolas Pouchelle, Modeling the detection of optical sights using retro reflection, Proc. SPIE 6950, forthcoming. 2. H. H. P. T. Bekman, J. C. van den Heuvel, F. J. M van Putten, and H. M. A Schleijpen, Development of a mid-infrared laser for directed infrared countermeasures, Proc. SPIE 5615, pp. 27-38, 2004.

3. H. M. A. Schleijpen, Johan C. van den Heuvel, Arjan L. Mieremet, Benoit Mellier, and Frank J. M. van Putten, Laser dazzling of focal plane array cameras, Proc. SPIE 6543, p. 65431B, 2007.

4. H. M. A. Schleijpen, S. R. Carpenter, B. Mellier, and A. Dimmeler, Imaging seeker surrogate for IRCM evaluation, Proc. SPIE 6397, p. 63970E, 2006.

5. H. M. A. Schleijpen, Alwin Dimmeler, Bernd Eberle, Johan C. van den Heuvel, Arjan L. Mieremet, Herman Bekman, and Benoit Mellier, Laser dazzling of focal plane array cameras, Proc. SPIE 6738, p. 67380O, 2007. 